\title{
Comparison of visual quality after EVO-ICL implantation and SMILE to select the appropriate surgical method for high myopia
}

Qin Qin ${ }^{1,2,3,4}$, Lianyun Bao², Liping Yang ${ }^{3,4}$, Zifang $\mathrm{He}^{2}$ and Zhenping Huang ${ }^{3,4^{*}}$ (D)

\begin{abstract}
Background: This study sought to compare the visual quality between intraocular collamer lens (EVO-ICL) implantation and small-incision lenticule extraction (SMILE) and determine the appropriate surgical method to treat patients with high myopia ( -6.25 to $-10 \mathrm{D})$.

Methods: A total of 48 eyes underwent EVO-ICL implantation and another 48 eyes underwent SMILE. The uncorrected distance visual acuity (UDVA), best-corrected distance visual acuity (BCVA) and equivalent spherical degree were compared across the SMILE (-6.25 to - 10 D) and EVO-ICL (- 6.25 to -10 D) implantation groups. Preoperative and postoperative visual quality parameters were compared between and within groups.

Results: The OQAS II values (OV 100\%) one week and one month after surgery and the modulation transfer function (MTF), OV 20\% and OV 9\% values one week after surgery in the SMILE group were lower than the respective preoperative values. The objective scatter index (OSI) value increased one week as well as one month after surgery compared with the preoperative values. The MTF cut-off value of the SMILE group was lower than that of the EVO-ICL implantation group three months after surgery.

Conclusions: For patients with high myopia, the postoperative visual quality of EVO- ICL implantation was slightly better than that of SMILE.
\end{abstract}

Keywords: Myopia, EVO intraocular collamer lens, Small-incision lenticule extraction

\section{Background}

Myopia refers to a situation in which the light through eyes is focused in front of the retina [1]. Due to the increasing high prevalence over the past few decades, myopia remains a significant public health issue in some areas of the world, especially East Asia [2]. Myopia is measured in dioptres and divided into four status groups (low, moderate, high and severe) based on the pathogenesis $[3,4]$. Although treatments including implantable collamer lens (ICL) and small-incision lenticule extraction

\footnotetext{
*Correspondence: hzp19633@126.com

${ }^{3}$ Department of Ophthalmology, Jinling Medical School of Nanjing Medical University, No. 305 East Zhongshan Road, Nanjing 210002, Jiangsu Province, China

${ }^{4}$ Department of Ophthalmology, Jinling Hospital, No. 305 East Zhongshan Road, Nanjing 210002, Jiangsu Province, China

Full list of author information is available at the end of the article
}

(SMILE) have been widely used to correct near-sightedness, the optimal surgical methods for patients with high myopia still remains controversial.

The Visian ICL ${ }^{\text {ma }}$ (STAAR Surgical, Nidau, Switzerland) is a posterior chamber phakic intraocular lens (IOL) [5-7]. Recently, a novel ICL operation based on an artificial hole (EVO Visian Implantable Collamer Lens) has been developed. Previous work shows that EVO-ICL implantation is satisfactory in terms of safety [8]. Moreover, EVO-ICL implantation is similar to the traditional ICL implantation with regard to inducing the higher-order aberrations and contrast sensitivity function [9]. Furthermore, the laser-assisted in situ keratomileusis (LASIK), major myopia corneal laser-assisted surgery, has been available for more than 30 years [10]. LASIK is accepted by both doctors and patients due to the

(c) The Author(s). 2019 Open Access This article is distributed under the terms of the Creative Commons Attribution 4.0 International License (http://creativecommons.org/licenses/by/4.0/), which permits unrestricted use, distribution, and 
accuracy and fast recovery in patients [11]. SMILE, also known as the flapless surgical approach, does not necessitate the lifting of the corneal flap. Because the risk of flap-related complications are reduced, SMILE may therefore have advantages over LASIK [12]. Furthermore, the early clinical outcomes of SMILE to correct myopia and myopic astigmatism are encouraging [13-18]. Even though the parameters for optical quality as well as intraocular scattering is valuable for the subsequent satisfaction and postoperative visual performance in myopia, the comparison between patients with SMILE and EVO-ICL implantation of these parameters is still unclear.

In the present study, the double-pass technique (via OQAS $^{\text {Tix }}$ II, Optical Analysis System, Visiometrics, Spain) was used to assess optical quality parameters and intraocular scattering in patients who had undergone EVO-ICL implantation or SMILE (Fig. 1). This comparative clinical study assessed the application scope of two types of surgical methods to treat high myopia. The findings of this study might help us to select the appropriate surgical methods for patients with high myopia.

\section{Methods}

\section{Patients}

In the first group, 48 eyes from 24 consecutive patients (13 women, 11 men; aged 20-34 years) who underwent the bilateral implantation of the posterior chamber phakic ICL with a $0.36 \mathrm{~mm}$ central artificial hole (EVO-ICL ${ }^{\mathrm{m}}$, STAAR Surgical) of myopia (manifest refraction spherical equivalent to -6.25 to -10.0 dioptres [D], manifest cylinder $<0.5 \mathrm{D}$, chamber depth $\geq 3.0 \mathrm{~mm}$, endothelial cells $\geq 2500 / \mathrm{mm}^{2}$ ) were assessed. The D stability of these patients was more than two years (an increase of <- $0.5 \mathrm{D}$ every year). Meanwhile, 48 eyes from 24 age-matched patients (12 women, 12 men; aged 2031 years) who underwent bilateral SMILE to correct myopia and myopic astigmatism (manifest refraction spherical equivalent of -6.25 to $-10 \mathrm{D}$, manifest cylinder of 0 to $1.00 \mathrm{D}$ ) of sufficient corneal thickness (estimated residual thickness of the stromal bed $>250 \mu \mathrm{m}$ ) at the Jinling Clinical Medical College of Nanjing Medical University and Nanjing Drum Tower Hospital Clinical College of Nanjing Medical University were enrolled in the current study. Patients with a history of ocular surgery, severe dry eye, progressive corneal degeneration, cataract or uveitis were excluded. Based on OCULYZERII (WaveLight, Alcon, Fort Worth, TX, USA), eyes with keratoconus were excluded because early keratoconus without clinical features could increase the security of corneal refractive surgery and prevent the occurrence of postoperative ectasia $[19,20]$. The institutional review board at Nanjing Medical University approved this study, which followed the tenets of the Declaration of Helsinki. Written informed consent was obtained from all patients after the nature and possible consequences of the study were explained.

\section{Operation procedure and follow-up period}

Before surgery, non-cycloplegic autorefraction, corneal topography, the uncorrected visual acuity (UCVA), the best-corrected visual acuity (BCVA), cycloplegic refraction, intraocular pressure, axial length, visual quality and scotopic pupil size were checked. Next, three mirror

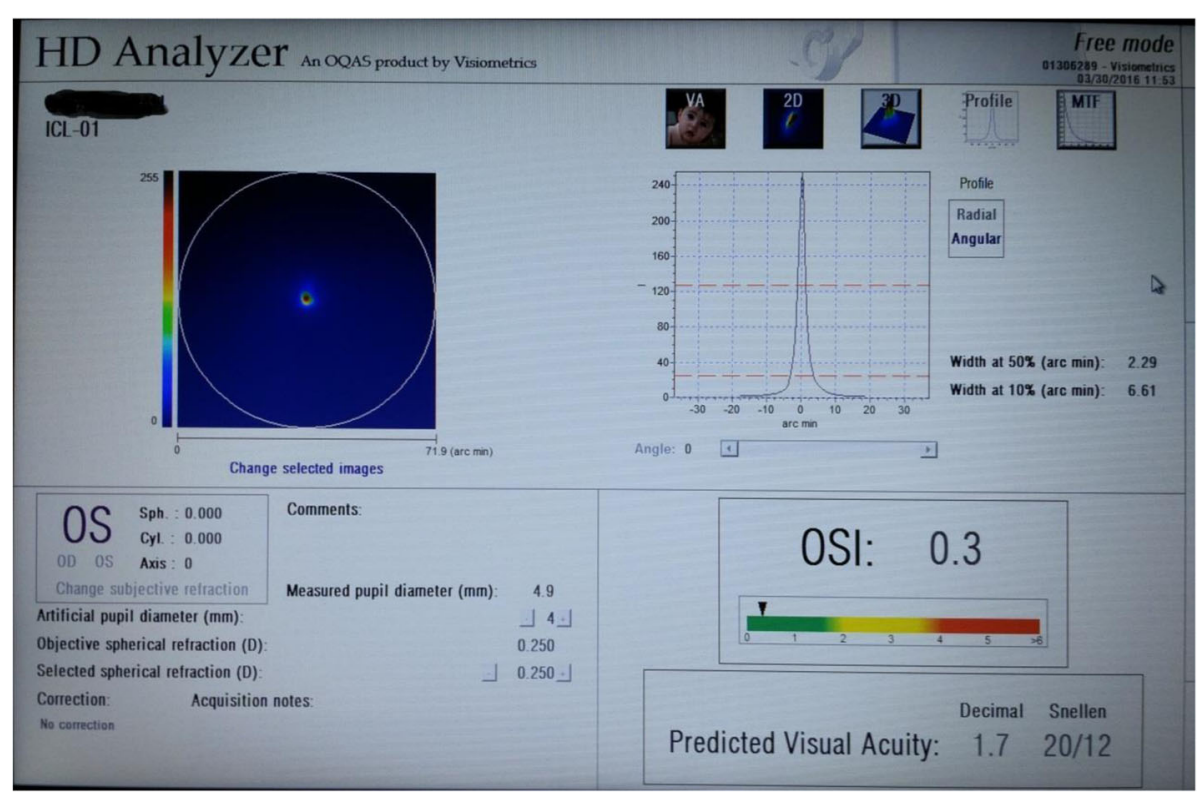

Fig. 1 OQAS II detection figure 
contact lenses were used to examine the retina. The laser photocoagulation treatment was administered for severe retinal degeneration or a torn hole. Anterior chamber depth, white-to-white distance, ciliary sulcus spacing and corneal endothelial cell counts were measured in the EVO-ICL implantation group. Antibiotic eye drops were used three times a day preoperatively. In this study, the same doctor was in charge of all surgeries.

\section{SMILE group}

After the surface anaesthesia with $0.4 \%$ oxybuprocaine, the VisuMax femtosecond laser system (Carl Zeiss, Meditec AG, Germany, $500-\mathrm{kHz}$ repetition rate) was performed on SMILE. A small (S) curved interface cone was used during each surgery. The anterior surface of the lenticule (spiral-out pattern) and the posterior surface of the lenticule (spiral-in pattern) were followed by a side cut of the cap. The power and spot distances for lenticule creation were $140 \mathrm{~nJ}$ power and $3.0 \mu \mathrm{m}$, respectively. Parameters for the femtosecond laser were $6.5 \mathrm{~mm}$ lenticule diameter, $120 \mu \mathrm{m}$ cap thickness, a 3 - $\mathrm{mm}$ side cut for the access to the lenticule with angles of $90^{\circ} 7.5 \mathrm{~mm}$ cap diameter and spot distance $2.0 \mu \mathrm{m}$ for a side cut. A spatula was inserted through the side cut over the roof of the refractive lenticule to dissect this plane, followed by the bottom of the lenticule. The lenticule was subsequently grasped with modified McPherson forceps (Geuder, GmbH, Heidelberg, Germany) and removed. After the removal of the lenticule, the intrastromal space was flushed with a balanced salt solution. After surgery, eye drops of tobramycin-dexamethasone (Tobradex, Alcon, Fort Worth, TX, USA) were administered into the eyes; this intervention was performed four times every day for one week. Flumetholon $(0.1 \%$ fluorometholone; Santen, Japan) was used four times a day for the second week; after which the frequency decreased by one administration per day each week for one month. Finally, 0.5\% antibiotic (levofloxacin; Santen, Japan) was administered topically four times every day for two weeks.

\section{EVO-ICL group}

The manufacturer performed the ICL power calculation (STAAR Surgical) by the modified vertex formula based on the ICL Power Calculation Software (http://en.infor mer.com/icl-power-calculation-software/, version 3.0). To decrease the preoperative refractive errors in each patient's eye, the target refraction was based on emmetropia. The panoramic ultrasound biomicroscope (UBM), which used to the study of anterior segment structures of human eye, could measure central corneal thickness (CCT), central anterior chamber depth (CACD) and anterior chamber angle etc. [21]. The manufacturer also decided the ICL size according to the parameter of horizontal corneal diameter, which was measured with a vernier caliper and the sulcus-to-sulcus (STS) distance using a panoramic UBM. We calculated the ideal ICL size as STS $+0.7 \mathrm{~mm}$. The anterior chamber depth and corneal curvature were measured with OCULYZERII (WaveLight, Alcon).

On the day of their surgery, patients were administered dilating and cycloplegic agents. After peribulbar anaesthesia, an EVO-ICL was inserted through a $3 \mathrm{~mm}$ clear corneal incision by the injector cartridge (STAAR Surgical) after the placement of hyaluronate (Shandong Bausch \& Lomb Freda Company) into the anterior chamber. After surgery, steroidal (1\% prednisolone acetate; Allergan, Ireland) and a $0.5 \%$ antibiotic (levofloxacin; Santen, Japan) was given four times every day for two weeks, after which the dose was decreased gradually.

\section{Measurement of visual quality}

After surgery, the optical quality and objective intraocular scattering measurements were performed with OQAS $^{\text {tw }}$ II (Optical Analysis System, Visiometrics, Spain) in a dark environment (approximately $25 \mathrm{~lx}$ ) preoperatively and at one week, one month and three months (for a $4.0 \mathrm{~mm}$ pupil). The device (OQAS II) has acceptable reliability, and the eye's realignment does not alter the measurements [22, 23]. The double-pass technique enables the assessment of the retinal image quality only with one specific pupil diameter per measurement; an additional measurement is required for other desired pupil sizes. Therefore, retinal image quality measures were assessed with a $4.0 \mathrm{~mm}$ pupil in this study. This standard size is often used to analyse ocular aberrations and it more closely simulates visual acuity measurements performed with an undilated pupil [24].

The instrument automatically corrected spherical ametropia between +6 and $-8 \mathrm{D}$. Ametropia beyond the spherical range or higher than the $0.5 \mathrm{D}$ cylinder required an additional lens on the instrument insert frame. The patient blinked a few times before each inspection to spread evenly over the tear film. The instrument was based on the double-pass technique to directly obtain a point-source retinal image analysis, and then the point spread function (PSF) was analysed. The objective visual analysis system of OQAS $\mathrm{II}^{\mathrm{Tm}}$ is based on the double-pass retinal imaging technique used in this study. The distribution of light intensity in the retina of the $780 \mathrm{~nm}$ infrared acquisition point-source imaging, with a description of the point-source resolution of the PSF of optical system expression analysis of intraocular optical imaging quality, and modulation transfer function was obtained by PSF. The optical quality and objective intraocular scattering parameters were analysed by PSF, including cut-off frequency of the modulation transfer function (MTF cut-off), Strehl ratio (SR), OQAS under 
different contrast value (OQAS values (OV) 100, 20, 9\%) and the objective scatter index (OSI). OQAS values under 100, 20 and 9\% contrast ratio are below the MTF cut-off frequency, and the 0.05 and 0.01 MTF values are segmented by $30 \mathrm{C} / \mathrm{deg}$., respectively.

\section{Statistical analysis}

SPSS 20.0 was used as the statistical software in the current study. UDVA, BCVA, spherical equivalent, optical quality, scattering function results, comparison of corneal endothelial cell density were compared between two groups. These parameters were also compared preand postoperatively within each group using generalised estimating equations tests. A $p$-value of $<0.05$ was considered significant.

\section{Results}

\section{Follow-up and baseline comparison}

No patients in this study were lost prior to the three-month follow-up. Our study found that differences in preoperative equivalent spherical D, UDVA and BCVA between the SMILE $(-6.25$ to $-10.0 \mathrm{D})$ and EVO-ICL $(-6.25$ to -10.0 D) groups were not significant (Table 1). None of the patients had obvious complications during either surgery. Some patients in the SMILE group exhibited transient haze during the early postoperative period. Ocular pain and corneal oedema were observed in two eyes with high intraocular pressure after EVO-ICL implantation. However, the symptoms disappeared $24 \mathrm{~h}$ after intravenous infusion of mannitol. The intraocular pressure in another patient was 28-31 $\mathrm{mmHg}(1 \mathrm{mmHg}=0.133 \mathrm{kPa})$ two weeks after surgery. Carteolol hydrochloride eye drops were administered twice per day, and the intraocular pressure recovered five days later $(17 \mathrm{mmHg})$. One month after surgery, the intraocular pressure was stable. In addition, none of the patients in the two groups exhibited serious

Table 1 Preoperative demographics of the eyes undergoing SMILE and EVO-ICL

\begin{tabular}{|c|c|c|c|}
\hline & $\begin{array}{l}\text { SMILE } \\
(-6.25 \mathrm{D} \sim-10 \mathrm{D})\end{array}$ & $\begin{array}{l}\text { EVO-ICL } \\
(-6.25 \mathrm{D} \sim-10 \mathrm{D})\end{array}$ & $P$ value \\
\hline Age(years) & 23 years $(20,31$ years $)$ & 24 years $(20,34$ years $)$ & \\
\hline Gender & Male: Female $=12: 12$ & Male:Female $=11: 13$ & \\
\hline Spherical & $-8.00 \pm 1.65$ & $-8.15 \pm 1.71$ & $P=0.689$ \\
\hline Equivalent (D) & $(-6.25 \sim-9.25)$ & $(-6.25 \sim-9.50)$ & \\
\hline LogMAR & $1.33 \pm 0.23(1.9 \sim 0.9)$ & $1.36 \pm 0.27(1.9 \sim 0.9)$ & $P=0.650$ \\
\hline \multicolumn{4}{|l|}{ UDVA } \\
\hline LogMAR & $-0.04 \pm 0.04(-0.1 \sim 0)$ & $-0.04 \pm 0.05(-0.1 \sim 0)$ & $P=0.763$ \\
\hline CDVA & & & \\
\hline
\end{tabular}

complications, such as epithelial implantation, infectious keratitis, corneal ectasia, endophthalmitis or lens opacity.

Safety and efficacy comparison between different groups All procedures showed acceptable safety and efficacy after surgery, and the patients did not experience BCVA loss. The safety indexes of the SMILE group and the EVO-ICL implantation group were $1.00 \pm 0.01$ and 1.01 \pm 0.02 , respectively. No significant difference was found between these two groups. The efficacy index of the SMILE group was $1.00 \pm 0.01$ and the EVO-ICL implantation group was $1.01 \pm 0.01$. Again, no significant difference was found between these two groups.

The postoperative visual acuity and refractive power among the different groups are shown in Table 2. First, a comparison within groups was performed: the UDVA in the SMILE and EVO-ICL implantation groups three months after surgery increased compared to preoperative UDVA values, and the difference was significant $(P<0.05)$. No significant difference was found between the preoperative and postoperative BCVA in the SMILE group $(P=0.318)$. The three-month postoperative BCVA values of the EVO-ICL implantation groups were higher than the preoperative values, and these differences were significant $(P=0.003)$. The equivalent spherical Ds three months after surgery in the two groups were lower than their respective preoperative values, and these differences were significant $(P=0.000)$.

The difference between UDVA, BCVA or the equivalent spherical $D$ values before the surgery or at any of the postoperative time periods between the SMILE and EVO-ICL implantation groups was not significant.

\section{Visual quality comparison before and after surgery in the SMILE and EVO-ICL groups}

The visual quality in the SMILE group before surgery as well as one week, one month and three months after surgery are listed in Table 3. Besides MTF at one week after surgery, no significant difference was found in the cut-off frequency or the SR in the SMILE group before surgery or at any time point after surgery. The OV 100\% values one week and one month after surgery and the MTF, OV 20\% and OV 9\% values one week after surgery were lower than their respective preoperative values $(P=0.040,0.048,0.011,0.002$ and 0.001 , respectively). The differences in OQAS values before surgery or at three months after surgery were not significant. After surgery, the OSI values at one week as well as one month were significantly higher than those before surgery $(P=0.000$ and 0.013 , respectively), and no significant difference was found in the OSI values before surgery and three months after surgery. 
Table 2 Time courses of the visual and refractive outcomes between the SMILE ( 6.25 to -10 D) and EVO-ICL ( -6.25 to -10 D) groups

\begin{tabular}{|c|c|c|c|c|c|}
\hline & \multirow[t]{2}{*}{ Preoperative } & \multicolumn{3}{|c|}{ Postoperative period } & \multirow[t]{2}{*}{$P$-value } \\
\hline & & 1 week & 1 month & 3 month & \\
\hline & LogMAR UDVA & & & & \\
\hline SMILE $(-6.25$ to -10 D) & $1.33 \pm 0.23$ & $-0.01 \pm 0.06$ & $-0.04 \pm 0.06$ & $-0.04 \pm 0.07$ & $P_{0}=0.000^{*}$ \\
\hline EVO-ICL $(-6.25$ to -10 D) & $1.36 \pm 0.27$ & $0.00 \pm 0.10$ & $-0.04 \pm 0.06$ & $-0.06 \pm 0.05$ & $P_{0}=0.000^{*}$ \\
\hline \multirow[t]{2}{*}{$P$-value } & $P_{1}=0.650$ & $P_{1}=0.448$ & $P_{1}=0.720$ & $P_{1}=0.207$ & \\
\hline & LogMAR BCVA & & & & \\
\hline SMILE $(-6.25$ to -10 D) & $-0.04 \pm 0.04$ & $-0.02 \pm 0.06$ & $-0.05 \pm 0.05$ & $-0.05 \pm 0.06$ & $P_{0}=0.318$ \\
\hline EVO-ICL $(-6.25$ to -10 D) & $-0.04 \pm 0.05$ & $-0.04 \pm 0.06$ & $-0.05 \pm 0.05$ & $-0.06 \pm 0.04$ & $P_{0}=0.003^{*}$ \\
\hline \multirow[t]{2}{*}{$P$-value } & $P_{1}=0.763$ & $P_{1}=0.324$ & $P_{1}=0.698$ & $P_{1}=0.345$ & \\
\hline & spherical equivalent(D) & & & & \\
\hline SMILE $(-6.25$ to -10 D) & $-6.00 \pm 1.68$ & $0.04 \pm 0.19$ & $0.00 \pm 0.21$ & $-0.06 \pm 0.31$ & $P_{0}=0.000^{*}$ \\
\hline EVO-ICL ( -6.25 to -10 D) & $-6.15 \pm 1.72$ & $0.03 \pm 0.20$ & $-0.01 \pm 0.20$ & $0.01 \pm 0.20$ & $P_{0}=0.000^{*}$ \\
\hline P-value & $P_{1}=0.189$ & $P_{1}=0.773$ & $P_{1}=0.892$ & $P_{1}=0.285$ & \\
\hline
\end{tabular}

$P_{0}=P$-value of the difference between the preoperative and three month values; $P_{1}=P$-value of the difference between the SMILE $(-6.25$ to $-10 \mathrm{D})$ and EVO-ICL implantation $(-6.25$ to $-10 \mathrm{D})$ groups; ${ }^{*} P<0.05=$ significant difference

LogMAR logarithm of the minimal angle of resolution, UDVA uncorrected distance visual acuity BCVA best-corrected distance visual acuity, $D$ diopter

The comparison of visual quality in the EVO-ICL implantation group before surgery as well as one week, one month and three months after surgery are listed in Table 4. The MTF, SR, OV 100\%, OV 20\% and OV $9 \%$ values one week after surgery were lower than their respective preoperative values $(P=0.000$, $0.010,0.002,0.000$ and 0.001 , respectively). The OSI values one week after surgery were higher than their respective preoperative values $(P=0.003)$. The

Table 3 Time courses of the optical quality parameters after SMILE

\begin{tabular}{|c|c|c|c|c|}
\hline & \multirow[t]{2}{*}{ Preoperative } & \multicolumn{3}{|c|}{ Postoperative period } \\
\hline & & 1 week & 1 month & 3 month \\
\hline $\begin{array}{l}\text { MTF cutoff } \\
\text { frequency (cpd) }\end{array}$ & \multirow[t]{2}{*}{$48.36 \pm 5.67$} & $44.81 \pm 7.33$ & $46.02 \pm 6.06$ & $46.34 \pm 4.23$ \\
\hline P-value & & $P_{1}=0.011^{*}$ & $P_{2}=0.126$ & $P_{3}=0.075$ \\
\hline SR & \multirow[t]{2}{*}{$0.25 \pm 0.05$} & $0.23 \pm 0.03$ & $0.25 \pm 0.06$ & $0.26 \pm 0.06$ \\
\hline P-value & & $P_{1}=0.916$ & $P_{2}=0.947$ & $P_{3}=0.953$ \\
\hline OV 100\% & \multirow[t]{2}{*}{$1.62 \pm 0.14$} & $1.45 \pm 0.33$ & $1.48 \pm 0.19$ & $1.54 \pm 0.16$ \\
\hline P-value & & $P_{1}=0.040^{*}$ & $P_{2}=0.048^{*}$ & $P_{3}=0.130$ \\
\hline OV 20\% & \multirow[t]{2}{*}{$1.26 \pm 0.23$} & $1.10 \pm 0.19$ & $1.12 \pm 0.03$ & $1.13 \pm 0.26$ \\
\hline P-value & & $P_{1}=0.002^{*}$ & $P_{2}=0.062$ & $P_{3}=0.085$ \\
\hline OV 9\% & \multirow[t]{2}{*}{$0.71 \pm 0.14$} & $0.60 \pm 0.09$ & $0.65 \pm 0.19$ & $0.67 \pm 0.13$ \\
\hline P-value & & $P_{1}=0.001^{*}$ & $P_{2}=0.553$ & $P_{3}=0.936$ \\
\hline OSI & \multirow[t]{2}{*}{$0.40 \pm 0.11$} & $0.55 \pm 0.22$ & $0.50 \pm 0.18$ & $0.42 \pm 0.13$ \\
\hline P-value & & $P_{1}=0.000^{*}$ & $P_{2}=0.013^{*}$ & $P_{3}=0.963$ \\
\hline $\begin{array}{l}P_{1}=P \text {-value of the } \\
\text { and one week aft } \\
\text { parameters before } \\
\text { difference in the } \\
\text { after surgery } \\
\text { MTF modulation tr } \\
{ }^{*} P<0.05=\text { signific }\end{array}$ & $\begin{array}{l}\text { ansfer function, } \\
\text { visual quality par } \\
\text { ant difference }\end{array}$ & e visual quality & parameters be & $\begin{array}{l}\text { ore surgery } \\
\text { visual quality } \\
\text { alue of the } \\
\text { ee months } \\
\text { OQAS value }\end{array}$ \\
\hline
\end{tabular}

difference in the OQAS values before surgery or one to three months after surgery were not significant.

\section{Visual quality comparison between two groups}

Table 5 compares the visual quality of patients with high myopia who received the two types of surgical methods. The visual quality of the SMILE and EVO-ICL groups was compared before surgery as well as one week and

Table 4 Time courses of the optical quality parameters after EVO-ICL

\begin{tabular}{lllll}
\hline & Preoperative & \multicolumn{3}{l}{ Postoperative period } \\
\cline { 3 - 5 } & & 1 week & 1 month & 3 month \\
\hline MTF cutoff & $48.46 \pm 5.18$ & $44.48 \pm 5.51$ & $47.17 \pm 10.23$ & $48.96 \pm 3.69$ \\
frequency (cpd) & & & & \\
$P$-value & & $P_{1}=0.000^{*}$ & $P_{2}=1.000$ & $P_{3}=1.000$ \\
SR & $0.26 \pm 0.06$ & $0.22 \pm 0.03$ & $0.24 \pm 0.08$ & $0.26 \pm 0.06$ \\
$P$-value & & $P_{1}=0.010^{*}$ & $P_{2}=0.749$ & $P_{3}=1.000$ \\
OV 100\% & $1.61 \pm 0.13$ & $1.45 \pm 0.29$ & $1.55 \pm 0.33$ & $1.59 \pm 0.17$ \\
P-value & & $P_{1}=0.002^{*}$ & $P_{2}=0.439$ & $P_{3}=1.000$ \\
OV 20\% & $1.22 \pm 0.21$ & $1.03 \pm 0.12$ & $0.12 \pm 0.31$ & $1.14 \pm 0.25$ \\
P-value & & $P_{1}=0.000^{*}$ & $P_{2}=0.621$ & $P_{3}=0.778$ \\
OV 9\% & $0.72 \pm 0.15$ & $0.61 \pm 0.09$ & $0.67 \pm 0.14$ & $0.69 \pm 0.16$ \\
$P$-value & & $P_{1}=0.001^{*}$ & $P_{2}=0.772$ & $P_{3}=1.000$ \\
OSI & $0.41 \pm 0.11$ & $0.54 \pm 0.26$ & $0.40 \pm 0.55$ & $0.43 \pm 0.13$ \\
P-value & & $P_{1}=0.003^{*}$ & $P_{2}=0.633$ & $P_{3}=1.000$ \\
\hline$P_{1}=P_{\text {-value }}$ & & &
\end{tabular}

$P_{1}=P$-value of the difference between visual quality parameters before surgery and one week after surgery; $P_{2}=P$-value of the difference between visual quality parameters before surgery and one month after surgery; $P_{3}=P$-value of the difference between visual quality parameters before surgery and three months after surgery

MTF modulation transfer function, OSI objective scattering index, OV OQAS value ${ }^{*} P<0.05=$ significant difference 
Table 5 Time courses of the optical quality parameters in the SMILE (-6.25 to -10 D) and EVO-ICL (-6.25 to -10 D) groups

\begin{tabular}{|c|c|c|c|c|c|c|}
\hline \multirow[t]{2}{*}{ MTF } & \multicolumn{2}{|l|}{ Preoperative } & \multicolumn{2}{|c|}{1 week postoperation } & \multicolumn{2}{|c|}{3 month postoperation } \\
\hline & $\overline{\text { SMILE }}$ & EVO-ICL & SMILE & EVO-ICL & SMILE & EVO-ICL \\
\hline Cut-off frequency & $48.36 \pm 5.67$ & $48.46 \pm 5.18$ & $44.81 \pm 7.33$ & $44.48 \pm 5.51$ & $46.34 \pm 4.23$ & $48.96 \pm 3.69$ \\
\hline$P$-value & \multicolumn{2}{|c|}{$P=1.000$} & \multicolumn{2}{|c|}{$P=1.000$} & \multicolumn{2}{|c|}{$P=0.049^{*}$} \\
\hline SR & $0.25 \pm 0.05$ & $0.26 \pm 0.06$ & $0.23 \pm 0.03$ & $0.22 \pm 0.03$ & $0.26 \pm 0.06$ & $0.26 \pm 0.06$ \\
\hline P-value & \multicolumn{2}{|c|}{$P=0.999$} & \multicolumn{2}{|c|}{$P=0.590$} & \multicolumn{2}{|c|}{$P=1.000$} \\
\hline OV $100 \%$ & $1.62 \pm 0.14$ & $1.61 \pm 0.13$ & $1.45 \pm 0.33$ & $1.45 \pm 0.29$ & $1.54 \pm 0.16$ & $1.59 \pm 0.17$ \\
\hline P-value & \multicolumn{2}{|c|}{$P=1.000$} & \multicolumn{2}{|c|}{$P=1.000$} & \multicolumn{2}{|c|}{$P=0.887$} \\
\hline OV 20\% & $1.26 \pm 0.23$ & $1.22 \pm 0.21$ & $1.10 \pm 0.19$ & $1.03 \pm 0.12$ & $1.13 \pm 0.26$ & $1.14 \pm 0.25$ \\
\hline$P$-value & \multicolumn{2}{|c|}{$P=1.000$} & \multicolumn{2}{|c|}{$P=0.359$} & \multicolumn{2}{|c|}{$P=1.000$} \\
\hline OV 9\% & $0.71 \pm 0.14$ & $0.72 \pm 0.15$ & $0.60 \pm 0.09$ & $0.61 \pm 0.09$ & $0.67 \pm 0.13$ & $0.69 \pm 0.16$ \\
\hline P-value & \multicolumn{2}{|c|}{$P=1.000$} & \multicolumn{2}{|c|}{$P=1.000$} & \multicolumn{2}{|c|}{$P=1.000$} \\
\hline OSI & $0.40 \pm 0.11$ & $0.41 \pm 0.11$ & $0.55 \pm 0.22$ & $0.54 \pm 0.26$ & $0.42 \pm 0.13$ & $0.43 \pm 0.13$ \\
\hline$P$-value & \multicolumn{2}{|c|}{$P=1.000$} & \multicolumn{2}{|c|}{$P=1.000$} & \multicolumn{2}{|c|}{$P=1.000$} \\
\hline
\end{tabular}

$P=P$-value of the difference between visual quality parameters in the SMILE and EVO-ICL implantation groups MTF modulation transfer function, OSI objective scattering index, OV OQAS value

${ }^{*} P<0.05=$ significant difference

three months after surgery. No significant differences were found in the MTF cut-off frequency, SR, OV 100\%, OV $20 \%$, OV $9 \%$ or OSI between the two groups at any time point. However, the MTF cut-off value in the SMILE group was lower than that in the EVO-ICL implantation group three months after surgery $(P=0.049)$.

Comparison of corneal endothelial cell density before and after surgery within and between the SMILE and EVO-ICL groups

Table 6 displays that there was no significant difference in corneal endothelial cell density (ECD) of SMILE (- 6.25 to $-10.0 \mathrm{D})$ and EVO-ICL $(-6.25$ to $-10.0 \mathrm{D})$ groups before and three months after surgery $(P>0.05)$. There was also no significant difference in the two time points between two groups $(P>0.05)$.

\section{Discussion}

The most common evaluation methods of visual quality after refractive surgery are subjective measurements of light and shade perception, environmental and contrast visual acuity as well as objective measurements of whole eye and corneal wavefront aberration [25-27]. However, scattering and diffraction are important factors that

Table 6 Comparison of corneal endothelial cell density within and between the SMILE(-6.25 to -10 D) and EVO-ICL (-6.25 to -10 D) groups

\begin{tabular}{lll}
\hline & Preoperative & 3 month Postoperative \\
\hline SMILE & $2875.88 \pm 99.82 / \mathrm{mm}^{2}$ & $2857.90 \pm 94.95 / \mathrm{mm}^{2}$ \\
EVO-ICL & $2876.17 \pm 102.49 / \mathrm{mm}^{2}$ & $2836.19 \pm 106.37 / \mathrm{mm}^{2}$ \\
\hline
\end{tabular}

Comparisons of ECD within and between the SMILE and EVO-ICL groups were all $P>0.05$ affect visual quality in humans. Femtosecond stromal interface quality was improved with a lower pulse energy and narrower spot separations than those currently used in clinical settings [28]. Kamiya [29] found that the remaining tissue islands in SMILE patients require mechanical dissection; results also indicated that the cavitation bubbles merge together and impair the subsequent laser beam, which results in an increase in surface irregularity. The results in this study showed that the visual quality of the SMILE group one week after surgery was lower than that before surgery. Actually, the femtosecond lenticule extraction led to a transient decrease in optical quality as well as an enhancement in the intraocular scattering during the early period of postoperative because of mild interface haze formation. The visual performance postoperatively after SMILE has been described as a certain extent due to the higher-order aberrations and visual acuity [12, 18, 30,31]. In addition, the recovery of visual acuity after SMILE is slightly slower than recovery after other techniques of keratorefractive surgery during the early postoperative period [32, 33]. Kamiya [34] assumed that this delay is caused by corneal scattering rather than higher-order aberrations resulting from surface irregularities, and that this is vital for visual performance after FLEx and SMILE. Similar to the previous study, our results indicated that OSI significantly increased one week after surgery and MTF, OV 100, 20\% and OV 9\% values decreased one week after SMILE. Therefore, we speculated that the decrease in visual quality one week after SMILE was related to the increase in corneal OSI and higher-order aberrations in corneal morphology changes and transient haze. Patients' visual quality was improved one month after surgery and was significantly improved 
three months after surgery. BCVA did not significantly differ before or after surgery perhaps because the haze regression led to improved visual quality.

A previous study [35] showed that compared with wavefront-guided LASIK, the ICL implantation has significantly fewer ocular higher-order aberrations not only in patients with high disease status but also in moderate or low disease status [36]. Buhren et al. [37] also reported that after implantation based on the artisan phakic IOL, the number of higher-order aberrations increased. The patients of the EVO-ICL implantation group in this study had less astigmatism; thus, the postoperative UDVA was less affected. The postoperative UDVA was better than the preoperative BCVA, and the MTF, SR,OV $100 \%$, OV $20 \%$ and OV 9\% values one week after the surgery were lower than their respective preoperative values. However, OSI values one week after surgery were higher than their respective preoperative values. The differences in the OQAS values before surgery or one and three months after surgery were not significant. We believe that the decrease of visual quality one week after surgery may be related to the early postoperative inflammatory response. To optimise ICL implantation visual performance, parameters such as increased higher-order aberrations and decreased retinal magnification might be useful and valuable [35, 36, 38-41]. In the present study, after ICL implantation, the excellent optical quality was in accordance with previous studies. Previous studies have suggested that the implantation of ICL increases the amount of intraocular refractive medium, which might lead to more intraocular scattering. Nevertheless, we hypothesise that ICL would not produce more intraocular scattering because the thickness of the EVO-ICL loop is $100-200 \mu \mathrm{m}$, the optical zone thickness is only $50-60 \mu \mathrm{m}$ and the ICL is located in the ciliary sulcus, which rarely tilts or shifts. Even the visual quality of patients with severe myopia and unhealthy fundus was partially improved.

With the increase in myopia, night glare and blurred vision, the visual quality problems caused by corneal aspheric changes increased spherical aberration and other higher-order aberrations after SMILE in patients with high myopia $[12,14,15,31]$. In addition, the corneal wound healing response, refractive regression [42] and the security problem make corneal refractive surgery a less attractive choice for patients with high myopia. However, patients with EVO-ICL implantation retained normal corneal morphology to avoid increasing cornea scattering and the increase in OSI and higher-order aberrations was lower. In the present study, the visual quality of the EVO-ICL implantation group and the SMILE group with high myopia did not show a significant difference except for the MTF value at three months after surgery. The results showed that SMILE and EVO-ICL might both achieve satisfactory, safe and effective postoperative visual quality in patients with high myopia. After the posterior chamber IOL implantation in the high myopia patients, the imaging of the external object in the retina was almost the same as that of emmetropic eyes. The retinal magnification of corneal surgery was 0.97, which was less than that of the posterior chamber IOL, which was close to that of the eye node, with a magnification of 1.0. Moreover, our results showed that there was no significant difference in corneal endothelial cell density (ECD) between and within the two groups before and three months after surgery. We speculated that the visual quality in patients with high myopia after EVO-ICL implantation was slightly better than SMILE three months after surgery. There were some limitations in this study. The optical parameters were assessed only for a $4.0 \mathrm{~mm}$ pupil. Additional research is necessary for confirmation of optical quality parameters under natural viewing conditions before and after surgery.

\section{Conclusions}

In conclusion, the postoperative visual quality after EVO-ICL implantation was a little better than that after SMILE in patients with -6.25 to $-10.0 \mathrm{D}$ myopia. If the corneal thickness is limited, patients with larger refractive regression may be expected. Therefore, EVO-ICL implantation is a better first choice. Meanwhile, the appropriate surgical procedure should be chosen based on the preoperative examination, which includes the thickness of the cornea, ACD, ECD etc. and the patient's own needs, such as financial status.

\section{Abbreviations \\ BCVA: Best-corrected distance visual acuity; ICL: Intraocular collamer lens; MTF: Modulation transfer function; OSI: Objective scatter index; OV: OQAS II values; UDVA: Uncorrected distance visual acuity \\ Acknowledgements \\ Not applicable \\ Funding \\ This work was supported by the Key Project, which was funded by the Medical Science and Technology Development Foundation, Nanjing Department of Health (YKK17078). The funding agencies had no role in the design or conduct of this study, including collection, management, analysis or interpretation of the data, preparation, review or approval of the manuscript or in the decision to submit the manuscript for publication.}

\section{Availability of data and materials}

The datasets used and/or analysed during the current study are available from the corresponding author on reasonable request.

\section{Authors' contributions}

QQ-Design, drafting, data acquisition, analysis and interpretation. LYB - Design, data acquisition and analysis. LPY_-Data acquisition, analysis and drafting. ZFH—Data acquisition, analysis and drafting. ZPH—Design, drafting, analysis and interpretation. All authors read and approved the final manuscript.

Ethics approval and consent to participate

The institutional review board at Nanjing Medical University approved this study, which followed the tenets of the Declaration of Helsinki. Written 
informed consent was obtained from all patients after the nature and possible consequences of the study were explained.

\section{Consent for publication}

Not applicable.

\section{Competing interests}

The authors declare that they have no competing interests.

\section{Publisher's Note}

Springer Nature remains neutral with regard to jurisdictional claims in published maps and institutional affiliations.

\section{Author details}

'Department of Ophthalmology, Nanjing Drum Tower Hospital Clinical College of Nanjing Medical University, Nanjing 210008, Jiangsu, China. ${ }^{2}$ Department of Ophthalmology, Nanjing Drum Tower Hospital, Affiliated to Nanjing University Medical School, Nanjing 210008, China. ${ }^{3}$ Department of Ophthalmology, Jinling Medical School of Nanjing Medical University, No. 305 East Zhongshan Road, Nanjing 210002, Jiangsu Province, China. ${ }^{4}$ Department of Ophthalmology, Jinling Hospital, No. 305 East Zhongshan Road, Nanjing 210002, Jiangsu Province, China.

\section{Received: 16 January 2018 Accepted: 4 January 2019}

\section{Published online: 08 February 2019}

\section{References}

1. Hornbeak DM, Young TL. Myopia genetics: a review of current research and emerging trends. Curr Opin Ophthalmol. 2009;20(5):356-62.

2. Morgan IG, Ohno-Matsui K, Saw SM. Myopia. Lancet. 2012;379(9827):1739.

3. Torky MA, Alzafiri YA. Visual and refractive outcomes of small-incision lenticule extraction in mild, moderate, and high myopia: six-month results. J Cataract Refract Surg. 2017;43(4):459-65.

4. Kim JR, Kim BK, Mun SJ, Chung YT, Kim HS. One year outcomes of small incision lenticule extraction: mild to moderate myopia versus high myopia. BMC Ophthalmol. 2015;10(15):59.

5. Alfonso JF, Baamonde B, Fernández-Vega L, Fernandes P, González-Méijome $J M$, Montés-Micó R. Posterior chamber collagen copolymer phakic intraocular lenses to correct myopia: five-year follow-up. J Cataract Refract Surg. 2011;37(5):873-80.

6. Igarashi A, Shimizu K, Kamiya K. Eight-year follow-up of posterior chamber phakic intraocular lens implantation for moderate to high myopia. Am J Ophthalmol. 2014;157(3):532-9.

7. Bloomenstein MR, Dulaney DD, Barnet RW, Perkins SA. Posterior chamber phakic intraocular lens for moderate myopia and hyperopia. Optometry. 2002;73(7):435-46.

8. Shimizu K, Kamiya K, Igarashi A, Shiratani T. Early clinical outcomes of implantation of posterior chamber phakic intraocular lens with a central hole (hole ICL) for moderate to high myopia. Br J Ophthalmol. 2012;96(3): 409-12.

9. Shimizu K, Kamiya K, Igarashi A, Shiratani T. Intraindividual comparison of visual performance after posterior chamber Phakic intraocular Lens with and without a central hole implantation for moderate to high myopia. Am J Ophthalmol. 2012;154(3):486-94.

10. Shortt AJ, Allan BD, Evans JR. Laser-assisted in-situ keratomileusis (LASIK) versus photorefractive keratectomy (PRK) for myopia. Cochrane Database Syst Rev. 2013;31(1):CD005135.

11. Wang L, Booth MA, Koch DD. Comparison of intraocular lens power calculation methods in eyes that have undergone laser-assisted in-situ keratomileusis. Trans Am Ophthalmol Soc. 2004;102:189-96.

12. Lin $F, X U Y$, Yang $Y$. Comparison of the visual results after SMILE and femtosecond laser-assisted LASIK for myopia. J Refract Surg. 2014;30(4): 248-54.

13. Sekundo W, Kunert KS, Blum M. Small incision corneal refractive surgery using the small incision lenticule extraction (SMILE) procedure for the correction of myopia and myopic astigmatism: results of a 6 month prospective study. Br J Ophthalmol. 2011;95(3):335-9.

14. Shah R, Shah S, Sengupta S. Results of small incision lenticule extraction: allin-one femtosecond laser refractive surgery. J Cataract Refract Surg. 2011; 37(1):127-37.
15. Shah R, Shah S. Effect of scanning patterns on the results of femtosecond laser lenticule extraction refractive surgery. J Cataract Refract Surg. 2011; 37(9):1636-47.

16. Riau AK, Ang HP, Lwin NC, Chaurasia SS, Tan DT, Mehta JS. Comparison of four different VisuMax circle patterns for flap creation after small incision lenticule extraction. J Refract Surg. 2013;29(4):236-44.

17. Kamiya K, Shimizu K, Igarashi A, Kobashi H. Visual and refractive outcomes of femtosecond lenticule extraction and small-incision lenticule extraction for myopia. Am J Ophthalmol. 2014;157(1):128-34.

18. Vestergaard AH, Grauslund J, Ivarsen AR, Hjortdal JØ. Efficacy, safety, predictability, contrast sensitivity, and aberrations after femtosecond laser lenticule extraction. J Cataract Refract Surg. 2014;40(3):403-11.

19. Bae GH, Kim JR, Kim CH, Lim DH, Chung ES, Chung TY. Corneal topographic and tomographic analysis of fellow eyes in unilateral keratoconus patients using Pentacam. Am J Ophthalmol. 2014;157(1):103-9.

20. Ruiseñor Vázquez PR, Galletti JD, Minguez N, Delrivo M, Fuentes Bonthoux F, Pförtner T, Galletti JG. Pentacam Scheimpflug tomography findings in topographically normal patients and subclinical keratoconus cases. Am J Ophthalmol. 2014;158(1):32-40.

21. Li D, Shu C. Evaluation of accuracy of anterior segment biological measurement by panoramic ultrasound imaging UBM. Chinese ophthalmic research. 2008;26(5):384-6.

22. Saad A, Saab M, Gatinel D. Repeatability of measurements with a double-pass system. J Cataract Refract Surg. 2010;36(1):28-33.

23. Vilaseca M, Peris E, Pujol J, Borras R, Arjona M. Intra- and intersession repeatability of a double-pass instrument. Optom Vis Sci. 2010;87(9):675-81.

24. Marcos S. Aberrations and visual performance following standard laser vision correction. J Refract Surg. 2001;17(5):S596-601.

25. Neeracher B, Senn P, Schipper I. Glare sensitivity and optical side effects 1 year after photorefractive keratectomy and laser in situ keratomileusis. J Cataract Refract Surg. 2004;30(8):1696-701.

26. Dougherty PJ, Bains HS. A retrospective comparison of LASIK outcomes for myopia and myopic astigmatism with conventional NIDEK versus wavefront-guided VISX and Alcon platforms. J Refract Surg. 2008;4(9): 891-6.

27. Prieto PM, Vargas-Martín F, Goelz S, Artal P. Analysis of the performance of the Hartmann-shack sensor in the human eye. J Opt Soc Am A Opt Image Sci Vis. 2000;17(8):1388-98.

28. Serrao S, Buratto L, Lombardo G, De Santo MP, Ducoli P, Lombardo M. Optimal parameters to improve the interface quality of the flap bed in femtosecond laser-assisted laser in situ keratomileusis. J Cataract Refract Surg. 2012;38(8):1453-9.

29. Kamiya K, Shimizu K, Igarashi A, Kobashi H. Effect of femtosecond laser setting on visual performance after small-incision lenticule extraction for myopia. Br J Ophthalmol. 2015;99(10):1381-7.

30. Jin HY, Wan T, Wu F, Yao K. Comparison of visual results and higher-order aberrations after small incision lenticule extraction (SMILE): high myopia vs. mild to moderate myopia. BMC Ophthalmol. 2017;17(1):118.

31. Sekundo W, Gertnere J, Bertelmann T, Solomatin I. One-year refractive results, contrast sensitivity, high-order aberrations and complications after myopic small-incision lenticule extraction (ReLEx SMILE). Graefes Arch Clin Exp Ophthalmol. 2014;252(5):837-43.

32. Moshirfar M, Shah TJ, Masud M, Linn SH, Ronquillo Y, Hoopes PC Sr. Surgical options for retreatment after small-incision lenticule extraction: Advantages and disadvantages. J Cataract Refract Surg. 2018:44(11):1384-89.

33. Kamiya K, Igarashi A, Ishii R, Sato N, Nishimoto H, Shimizu K. Early clinical outcomes, including efficacy and endothelial cell loss, of refractive lenticule extraction using a $500 \mathrm{kHz}$ femtosecond laser to correct myopia. J Cataract Refract Surg. 2012;38(11):1996-2002.

34. Kamiya K, Shimizu K, Igarashi A, Kobashi H. Time course of optical quality and intraocular scattering after refractive Lenticule extraction. PLoS One. 2013;8(10):e76738

35. Igarashi A, Kamiya K, Shimizu K, Komatsu M. Visual performance after implantable collamer lens implantation and wavefront-guided laser in situ keratomileusis for high myopia. Am J Ophthalmol. 2009;148(1):164-70

36. Kamiya K, Igarashi A, Shimizu K, Matsumura K, Komatsu M. Visual performance after posterior chamber phakic intraocular lens implantation and wavefrontguided laser in situ keratomileusis for low to moderate myopia. Am J Ophthalmol. 2012;153(6):1178-86.

37. Sanders DR, Vukich JA, Doney K, Gaston M. Implantable contact Lens in treatment of myopia study Group.U.S. Food and Drug Administration 
clinical trial of the implantable contact Lens for moderate to high myopia. Ophthalmology. 2003;110(2):255-66.

38. Kamiya K, Shimizu K, Kawamorita T, Uozato H. Effects of myopic correction by replacement of spectacles, laser in situ keratomileusis, and phakic intraocular lens implantation on modulation transfer function and retinal magnification. Nippon Ganka Gakkai Zasshi. 2008;112(6):519-24.

39. Applegate RA, Howland HC. Magnification and visual acuity in refractive surgery. Arch Ophthalmol. 1993;111(10):1335-42.

40. Applegate RA, Chundru U. Experimental verification of computational methods to calculate magnification in refractive surgery. Arch Ophthalmol. 1995;113(5):571-7.

41. García M, González C, Pascual I, Fimia A. Magnification and visual acuity in highly myopic phakic eyes corrected with an anterior chamber intraocular lens versus by other methods. J Cataract Refract Surg. 1996;22(10):1416-22.

42. Ganesh S, Gupta R. Comparison of visual and refractive outcomes following femtosecond laser- assisted lasik with smile in patients with myopia or myopic astigmatism. J Refract Surg. 2014;30(9):590-6.

Ready to submit your research? Choose BMC and benefit from:

- fast, convenient online submission

- thorough peer review by experienced researchers in your field

- rapid publication on acceptance

- support for research data, including large and complex data types

- gold Open Access which fosters wider collaboration and increased citations

- maximum visibility for your research: over $100 \mathrm{M}$ website views per year

At $\mathrm{BMC}$, research is always in progress.

Learn more biomedcentral.com/submissions 